OPEN ACCESS

Edited by:

Swee-Suak Ko,

Academia Sinica, Taiwan

Reviewed by:

Rita Sharma,

Jawaharlal Nehru University, India

Sailendra Nath Sarkar,

University of Calcutta, India

${ }^{*}$ Correspondence:

Rui Wang

wangray1987@163.com

Specialty section:

This article was submitted to

Plant Biotechnology,

a section of the journal

Frontiers in Plant Science

Received: 18 July 2017 Accepted: 21 November 2017 Published: 06 December 2017

Citation:

Liu J, Gao F, Ren J, Lu X, Ren G and Wang $R$ (2017) A Novel AP2/ERF

Transcription Factor CR1 Regulates the Accumulation of Vindoline and Serpentine in Catharanthus roseus. Front. Plant Sci. 8:2082. doi: 10.3389/fp/s.2017.02082

\section{A Novel AP2/ERF Transcription Factor CR1 Regulates the Accumulation of Vindoline and Serpentine in Catharanthus roseus}

\author{
Jiaqi Liu, Fangyuan Gao, Juansheng Ren, Xianjun Lu, Guangjun Ren and Rui Wang* \\ Crop Research Institute, Sichuan Academy of Agricultural Sciences, Chengdu, China
}

As one type of the most important alkaloids in the world, terpenoid indole alkaloids (TIAs) show a wide range of pharmaceutical activities that are beneficial for clinical treatments. Catharanthus roseus produces approximately 130 identified TIAs and is considered to be a model plant to study TIA biosynthesis. In order to increase the production of high medical value metabolites whose yields are extremely low in C. roseus, genetic engineering combined with transcriptional regulation has been applied in recent years. By using bioinformatics which is based on RNA sequencing (RNA-seq) data from methyl jasmonate (MeJA)-treated C. roseus as well as phylogenetic analysis, the present work aims to screen candidate genes that may be involved in the regulation of TIA biosynthesis, resulting in a novel AP2/ERF transcription factor, CR1 (Catharanthus roseus 1). Subsequently, virus-induced gene silencing (VIGS) of CR1 was carried out to identify the involvement of CR1 in the accumulations of several TIAs and quantitative real-time PCR (qRT-PCR) was then applied to detect the expression levels of 7 genes in the related biosynthetic pathway in silenced plants. The results show that all the 7 genes were upregulated in CR1-silenced plants. Furthermore, metabolite analyses indicate that silencing CR1 could increase the accumulations of vindoline and serpentine in C. roseus. These results suggest a novel negative regulator which may be involved in the TIAs biosynthetic pathway.

Keywords: terpenoid indole alkaloids, RNA-seq, phylogenetic analysis, AP2/ERF, VIGS, qRT-PCR, metabolite analysis, Catharanthus roseus

\section{INTRODUCTION}

Plants produce a vast number of secondary metabolites, when they adapt themselves to variable ecological environment during long-term evolution. Secondary metabolites are usually derived from primary metabolites and have their own unique metabolic pathways and different properties. They are generally classified into three major groups: phenols, terpenoids and alkaloids. As one type of the most important alkaloids in the world, terpenoid indole alkaloids (TIAs) show a wide range of pharmaceutical activities that are beneficial for clinical treatments (Van Der Heijden et al., 2004; Wasternack, 2014). Most TIAs are produced from the plants of Apocynaceae, Loganiaceae and Rubiaceae. One from Apocynaceae, Catharanthus roseus, produces approximately 130 identified TIAs and is considered to be a model plant to study TIA biosynthesis. Most of the TIAs produced by C. roseus, such as ajmaline, serpentine and catharanthine, show significant clinical medical values, 
moreover, vinblastine and vincristine, which are recognized to be the most valuable antineoplastic agents, have been widely used in the world. However, the yields of these high medical value metabolites are extremely low (Guéritte and Fahy, 2005).

During a long time, chemical synthesis, plant tissue, microbiological culture and other efforts have been done to increase the production of valuable secondary metabolites. But these methods still need to be optimized because of the excessive costs and low production. In recent years, genetic engineering has been applied in an attempt to increase TIAs yields, for example, transcriptionally regulating some specific genes in hairy root or cell suspension cultures to change the accumulations of specific TIAs (Papon et al., 2004; Runguphan et al., 2009; Peebles et al., 2011; Cui et al., 2015), while few great break-through have been made through these strategies. The major reasons could be: (1) few candidate genes involved in the accumulation of TIAs have been identified; (2) the limited effects on comprehensive TIAs synthetic pathway by single candidate gene. Hence, it is critical to find new candidate genes which could greatly facilitate TIAs production and the researches of TIA biosynthesis regulation.

Lots of studies suggest that genes in the TIAs synthetic pathway are under tight transcriptional regulations. It has been revealed that transcription factors are able to increase the production of TIAs via regulating the expression of multiple genes in the synthetic pathway. ORCA2 and ORCA3 are firstly identified AP2/ERF transcription factors in C. roseus which are involved in the TIAs synthetic pathway (Menke et al., 1999; Van der Fits and Memelink, 2000). Overexpressing either ORCA2 or ORCA3 in C. roseus suspension cells or hairy roots could change the expression of several key genes in the TIAs biosynthetic pathway (Van der Fits and Memelink, 2000; Pan et al., 2012; Li et al., 2013; Sun and Peebles, 2016). In addition, ORCA3 could be regulated by many inducers, such as jasmonic acid, artemisinic acid and fungal endophytes (Peebles et al., 2009; Pandey et al., 2016; Wang et al., 2016). Another two AP2/ERF members, ORCA4 and ORCA5, were cloned recently. They formed a cluster with ORCA3 and also can be induced by jasmonic acid, ORCA4 could act on the transcripts levels of genes in both tryptophan pathway and seco-iridoid pathway (Paul et al., 2016). In 2011, CrMYC2 in bHLH (basic helix-loop-helix) family, isolated by a yeast one-hybrid screening, could regulate the expression of ORCAs and also had a strong effect on the accumulation of catharanthine and tabersonine (Montiel et al., 2011; Zhang et al., 2011). WRKY transcription factor CrWRKY1 regulates several key genes in the TIAs synthetic pathway, especially TDC (tryptophan decarboxylase). Overexpressing CrWRKY1 in the hairy root of $C$. roseus caused significantly increased accumulation of serpentine (Suttipanta et al., 2011; Yang et al., 2013).

The genomic and transcriptomic information of $C$. roseus are not completely uncovered as many model plants like tobacco, Arabidopsis and rice. Hence, the studies aiming to excavate and functionally identify important transcription factors in C. roseus are not comprehensive. RNA sequencing is a widespread high-throughput technology in recent years (Mortazavi et al., 2008), which offers a holistic view of the transcriptome expression profiles of selected plant tissues or cells and provides an efficient way to identify transcripts involved in specific biological processes (Cloonan et al., 2008; Wilhelm et al., 2010). Therefore, excavating relative transcription factors combined with transcriptome sequencing data and understanding of the mechanism of transcriptional regulation during the overall TIAs biosynthetic pathway may promote our ability to break through the synthetic bottleneck (Aito et al., 2008; Saito and Matsuda, 2010; Guo et al., 2013; Verma et al., 2014; Yang et al., 2017).

In this study, three candidate AP2/ERF transcription factors were selected based on transcriptome sequencing data from C. roseus with methyl jasmonate (MeJA) treatment, and their responses to MeJA were verified by quantitative real-time PCR (qRT-PCR). After phylogenetic tree analysis of the three candidate genes with known AP2/ERF transcription factors involved in secondary metabolite biosynthetic pathway, CR1, which forms a cluster with OpERF2, was chosen for further study. Subsequently, Virus-induced gene silencing (VIGS) of CR1 combined with metabolite analyses of silenced plants was performed to identify the involvement of CR1 in the accumulations of several TIAs. The results show that silencing CR1 could increase the accumulations of vindoline and serpentine in C. roseus.

\section{MATERIALS AND METHODS}

\section{Plant Growth Conditions and RNA-Seq}

Seeds of $C$. roseus cv. Rose Red were germinated in a greenhouse at $28^{\circ} \mathrm{C}, 16 / 8 \mathrm{~h}$ photoperiod on MS plates. After 4 weeks, seedlings with two to three pairs of true leaves were transferred to soil. Samples were collected from 1-month-old plants which were treated with MeJA for 0,1 or $4 \mathrm{~h}$. The MeJA experiment was with three replicates. Three plants were combined from each replicate time sample. MeJA treatment consisted of spraying $100 \mu \mathrm{M}$ MeJA then placing plants under a clear plastic dome sealed with tape. Leaves of the plants were harvested and then frozen in liquid nitrogen.

Total RNA extracted from each material were subjected to cDNA library preparation and sequenced at Novogene Bioinformatics Institute (China) on Illumina HiSeq ${ }^{\mathrm{TM}} 2000$ sequencer (Illumina Inc., United States). Clean reads were aligned based on the reference genome (Medicinal Plant Genomics Resource, MPGR) (Kellner et al., 2015), transcript abundant estimation and expression level in fragment per kilobase exon per million mapped fragment (FPKM) were performed as described by Chen et al. (2017).

\section{Phylogenetic Analysis}

Full-length amino acid sequences of candidate transcription factor as well as the AP2/ERFs which are potentially involved in secondary metabolite biosynthesis were aligned by ClustalW program. The neighbor-joining phylogeny was generated using MEGA 6 with bootstrap analysis of 1,000 replicates.

\section{Virus-Induced Gene Silencing}

Seeds of $C$. roseus cv. Rose Red were germinated in a greenhouse at $28^{\circ} \mathrm{C}, 16 / 8 \mathrm{~h}$ photoperiod. Four weeks later, plants with 
two to three pairs of true leaves were used for VIGS. The genomic DNA of $C$. roseus $\mathrm{cv}$. Rose Red was used as template to amplify CR1 with specific primers (Table 1). The PCR-generated fragment was then introduced into the terminal vector pTRV2 by $B a m \mathrm{H}$ I and Xho I digestion. Catharanthus phytoene desaturase (PDS) involved in chlorophyll biosynthesis produces visible photo bleaching and serves as a useful marker for determining the best time to collect samples for testing. The purified plasmid DNA pTRV2-CR1, pTRV2 (negative control), pTRV2-PDS were respectively transformed into Agrobacterium tumefaciens strain GV3101 by electroporation. Three kinds of strains and GV3101 harboring pTRV1 were cultured overnight at $28^{\circ} \mathrm{C}$ in $300 \mathrm{ml}$ Luria-Bertani medium containing $10 \mathrm{Mm}$ MES, $20 \mu \mathrm{M}$ acetosyringone and $50 \mu \mathrm{g} / \mathrm{mL}$ kanamycin. These bacterial pellets were collected and then resuspended in $5 \mathrm{ml}$ infiltration buffer (10 mM MES, $200 \mu \mathrm{M}$ acetosyringone and $1 \mathrm{mM} \mathrm{MgCl}_{2}$ ), and further incubated at $28^{\circ} \mathrm{C}$ for $3 \mathrm{~h}$ with shaking. The suspension of strain harboring pTRV1 was mixed with pTRV2-CR1, pTRV2 or pTRV2-PDS in equal volume just before the infection. The leaves of the seedlings were injected by a needle less syringe to fill the whole leaf with the bacteria, and each seedling was injected with 2-4 leaves. After injection, the plants were cultured in a constant temperature incubator. The PDS phenotype was observed 3 weeks after inoculation of leaves, and the other two sample plants were harvested at this stage. After recording the fresh weights of harvested materials, the samples were frozen in liquid nitrogen, one member of a leaf pair was used for RNA extraction, while the other was used for metabolite analysis.

\section{Expression Analysis by Quantitative Real-Time PCR}

Total RNAs of CR1-silenced plants and control were extracted and reverse-transcribed as described previously (Wang et al., 2015), and qRT-PCR was performed according to the same reference. The primers of CR1, CR2 and CR3 as well as 7 genes whose expression levels were detected in our work are shown in Table 1. Relative expression levels were determined using $2^{-\Delta \Delta C_{\mathrm{T}}}$ method (Livak and Schmittgen, 2001). All qRT-PCR analyses were performed with three independent biological replicates.

\section{Metabolite Analyses}

The selected samples were quickly pulverized in liquid nitrogen. Precisely weighed $0.3 \mathrm{~g}$ powder, mixed with $2 \mathrm{~mL}$ methanol, then subjected to extraction using the ultrasonic for $20 \mathrm{~min}$, the supernatant was obtained after centrifugation. Repeatedly extracted with methanol for three times, then combined supernatant extract and compressed to dry, diluted to $2 \mathrm{~mL}$ with methanol, finally, filtrated with $0.45 \mu \mathrm{m}$ microporous membrane before metabolite analyses by MS. The three standards catharanthine, vindoline and vinblastine were configured as a reserve solution of $10 \mathrm{ng} / \mathrm{L}$ with methanol, stored at $-20^{\circ} \mathrm{C}$,

TABLE 1 | Polymerase chain reaction (PCR) primers used in this study.

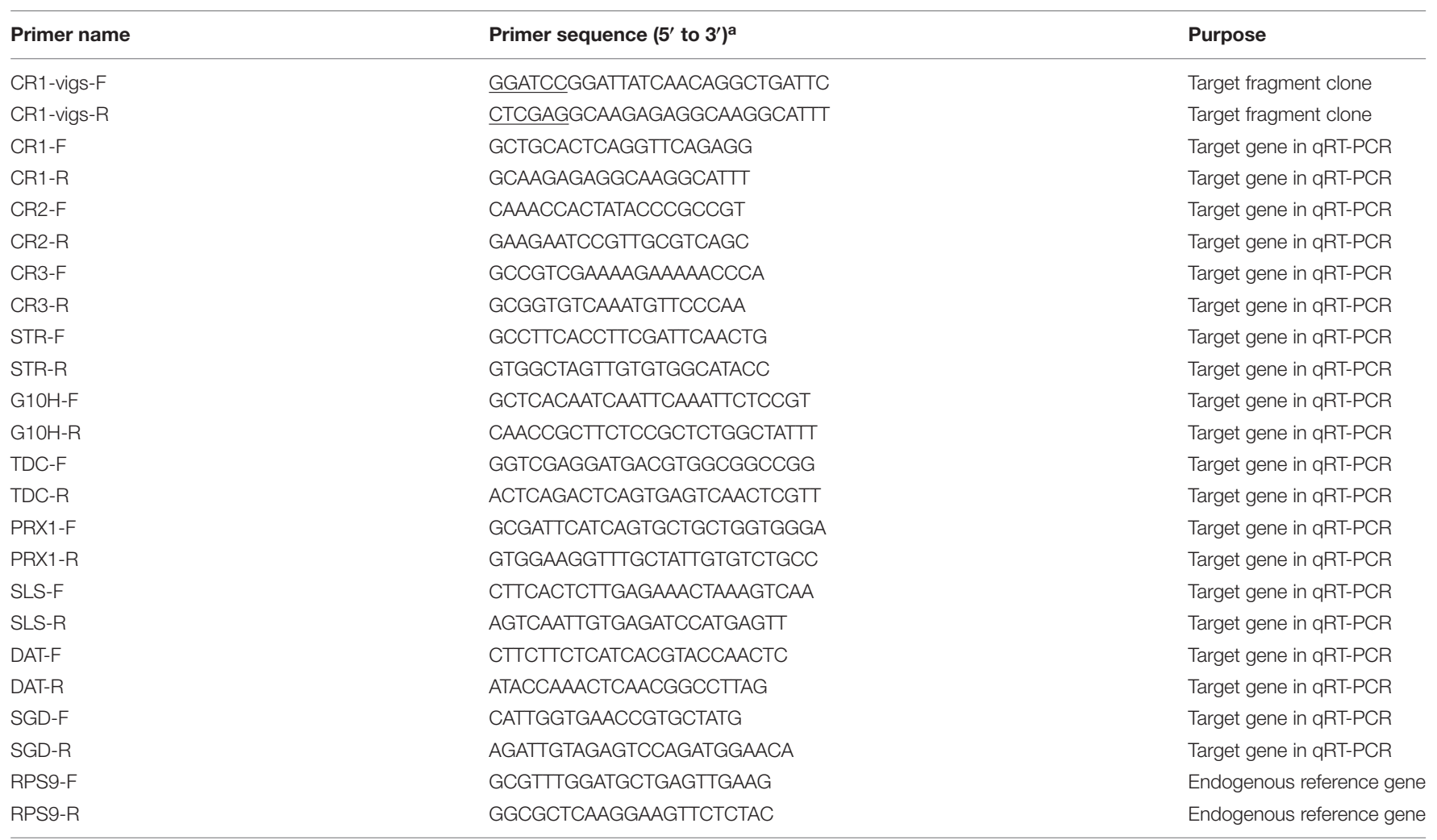

aThe underlined letters indicate the restriction enzyme sites. 


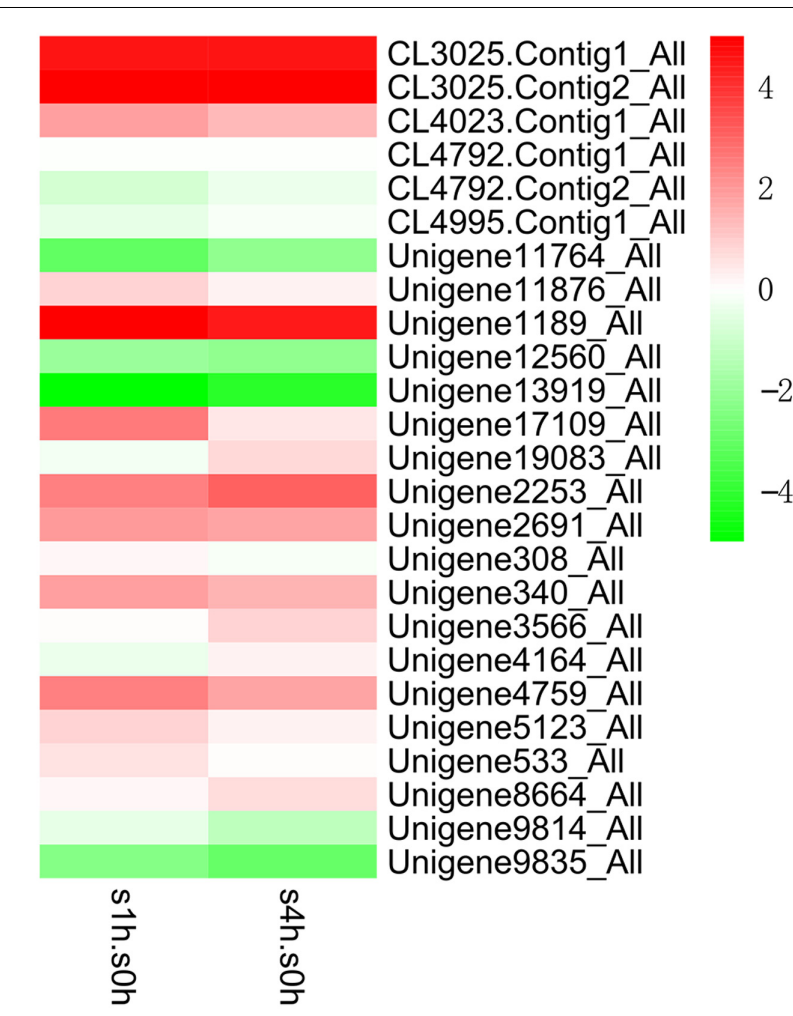

FIGURE 1 | Expression patterns ( $\log _{2}$ of FPKM) of AP2/ERF family in C. roseus at 1-h (s1h.sOh) and 4-h (s4h.sOh) after MeJA treatment. The expression levels are illustrated in green-red scale shown on the right. Green indicates lower expression and red indicates higher expression. Figure taken from Liu et al. (2017).

diluted to $10 \mathrm{ng} / \mathrm{mL}$ working fluid at the same time for testing purposes. ESI/MS/MS was used to detect the catharanthine, vindoline and vinblastine; using time-of-flight mass spectrometry (TOF-MS) to detect the serpentine.

\section{RESULTS}

\section{Three Candidate AP2/ERF Genes Were Selected Based on RNA-seq Data}

MeJA is identified as an important signaling molecule in TIA biosynthetic pathway in many plants (De Geyter et al., 2012). In order to excavate candidate transcription factors that may be involved in the regulation of TIA biosynthesis, we collected the leaves of 1-month-old C. roseus with MeJA treatment and extracted their RNA to generate the transcriptome sequencing data using Illumina platform. Subsequently, the expression profiles of different transcription factor families in $C$. roseus after MeJA treatment were analyzed. The expression profile of AP2/ERF family indicated that the expression levels of many AP2/ERF genes were changed obviously at 1 -h and 4-h after MeJA treatment (their annotated gene names are shown in Supplementary Table S1). Three candidate AP2/ERF genes with different representative response to MeJA were selected for further studies (Figure 1). The expression level of Unigene9835_ALL (here designated as CR1) gradually decreased along the treatment. The expression level of Unigene2253_ALL (here designated as CR2) gradually increased after MeJA treatment. The transcript of Unigene1189_All (here designated as CR3) was up-regulated dramatically at 1 -h and 4 -h after MeJA treatment, while the expression level at 4-h was slightly lower than at $1-\mathrm{h}$. The expression pattern suggested that CR3 is sharply induced by MeJA in a short time, and its expression level might gradually restore to the initial level. Above genes show different responses to MeJA treatment, and may be involved in the regulation of TIAs synthesis in $C$. roseus.

In order to determine the accuracy of the RNA-seq data, the expression levels of three candidate genes at different time point after MeJA treatment were confirmed by qRT-PCR. Figure 2 shows that after MeJA treatment, the expression level of CR1 decreased gradually with time; CR2 showed gradually increased transcript along MeJA treatment; the expression level of CR3 was up-regulated rapidly and reached a peak at $2-\mathrm{h}$, then gradually declined. According to the results, expression patterns of three candidate genes under MeJA treatment were consistent with the RNA-seq data. Hence, the above candidate genes were subjected to further study.

\section{Phylogenetic Analysis}

A phylogenetic tree was constructed based on the protein sequences of the three candidate transcription factors (for their sequences, see Supplementary Table S2), together with the previously reported AP2/ERF transcription factors which are potentially involved in secondary metabolite biosynthesis, to analyze their evolutionary relationships (Figure 3). CR1 was classified to the same group with CR2, CR3 and OpERF2. Besides, CR1 showed the closest relationship with OpERF2 which was identified recently to be involved in the regulation of specialized metabolism in Ophiorrhiza pumila (Udomsom et al., 2016), while CR2 and CR3 form the same cluster. The divergent clusters as well as the opposite response to MeJA between CR1 and CR2 or CR3 suggest the different molecular functions between CR1 and the other two. ORCA2 and ORCA3 involved in MIA biosynthesis in C. roseus; NbERF1, NtERF189 and NtORC1 regulating nicotine alkaloids biosynthesis; AaERF1, AaERF2 and AaORA regulating terpenoid biosynthesis in Artemisia annua, all of them showed more distant relationship with three candidate transcription factors than OpERF2 (Van der Fits and Memelink, 2001; Rushton et al., 2008; Shoji et al., 2010; Todd et al., 2010; De Boer et al., 2011; Yu et al., 2012; Lu et al., 2013; Thagun et al., 2016). Since CR1 showed a close phylogenetic relationship with other secondary metabolite-regulating AP2/ERFs, which suggested that CR1 may be a potential regulator in TIA biosynthesis, we selected CR1 for further functional characterization.

\section{CR1 Negatively Regulates 7 Key Genes in the TIAs Synthetic Pathway}

Virus-induced gene silencing is a technique used for functional analysis of genes, which can be effectively applied in plants 

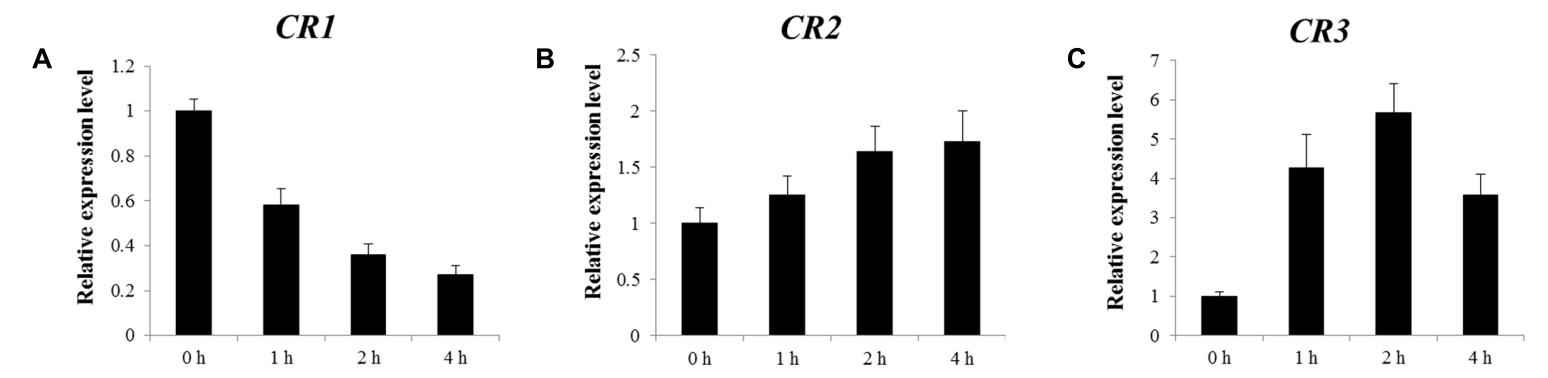

FIGURE 2 | Relative expression levels of CR1 (A), CR2 (B), and CR3 (C) responding to MeJA treatment. Figure taken from Liu et al. (2017).

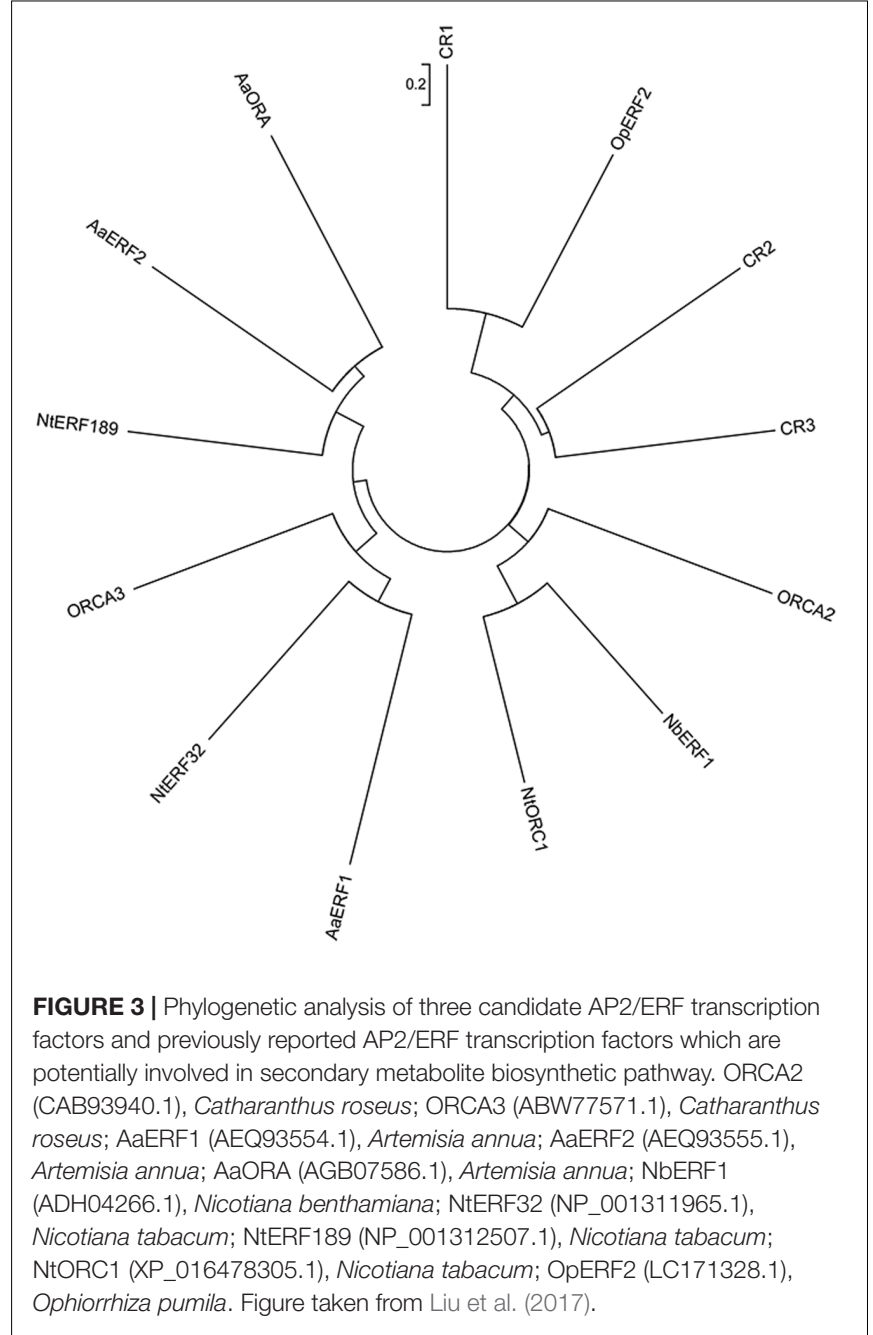

with long growth cycle and low conversion efficiency. The utility of this approach has been successful in exploring new genes that were involved in iridoid biosynthesis (Geu-Flores et al., 2012) and MIA biosynthesis (Liscombe and O'Connor, 2011) in C. roseus. In order to identify the function of CR1, we selected VIGS technology with the pTRV vector system (Dinesh-Kumar et al., 2003; Burch-Smith et al., 2004; Velásquez et al., 2009).

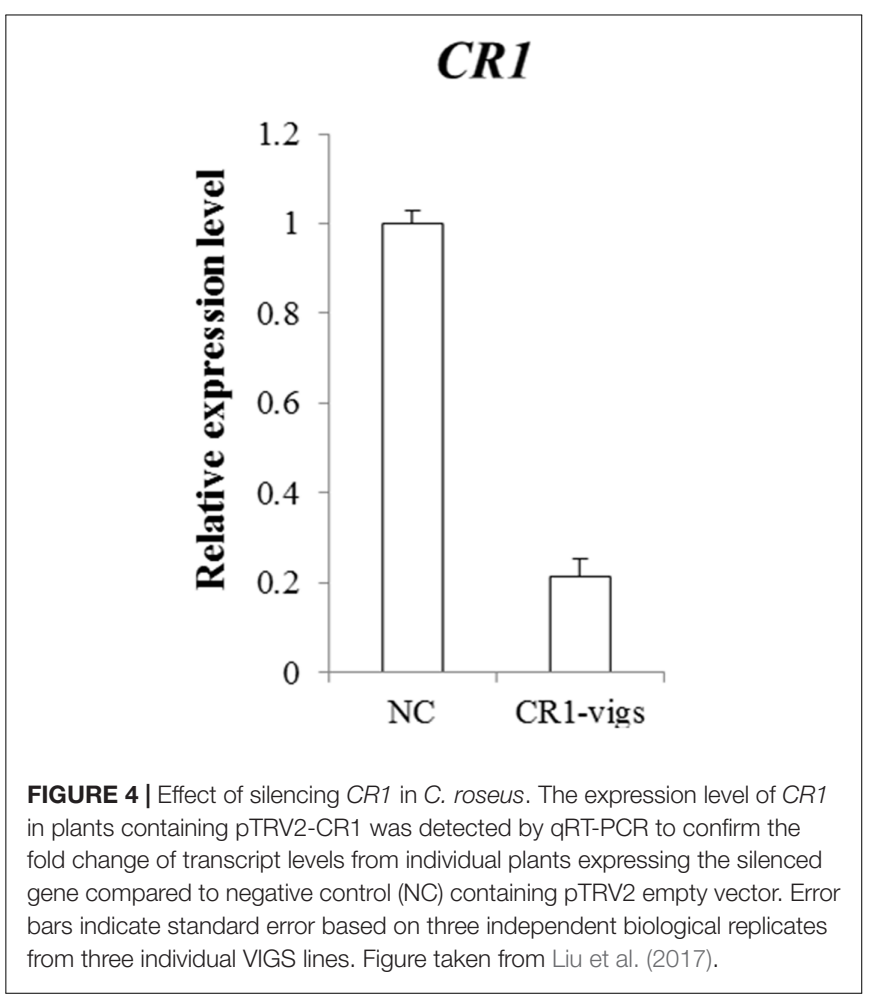

Transcript analysis of CR1 monitored by qRT-PCR revealed that the transcript level decreased by approximately $78 \%$ in CR1-silenced plants compared with the negative control (Figure 4). Subsequently, expression analysis of 7 genes $(\mathrm{G} 10 \mathrm{H}$, SLS, TDC, STR, SGD, DAT and PRX) which are representative in the TIAs synthetic pathway (Figure 5) in CR1-silenced plants was carried out. Their GenBank accession numbers are as follows: KF561461.1, L10081.1, X67662.1, X53602.1, EU072423.1, AF053307.1 and AM236087.1. The results indicated that silencing CR1 in C. roseus could result in obvious changes in the expression levels of these genes. The expression levels of G10H, SLS, TDC, STR, SGD, DAT and PRX showed significant up-regulation (Figure 6). According to the previous results which suggest that the expression level of $C R 1$ is down-regulated in response to MeJA treatment, it can be inferred that CR1 may be a new negative regulatory transcription factor in the pathway of TIA biosynthesis. 


\section{Geranyl diphosphate (GPP)}

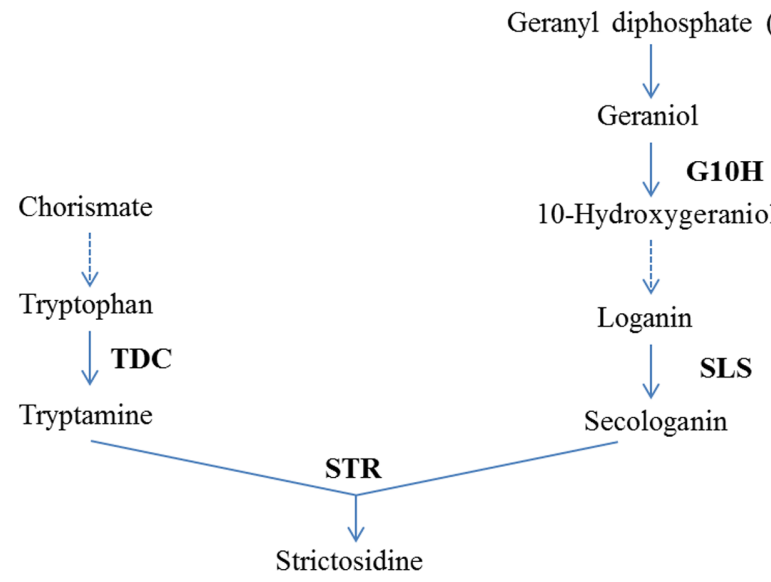

SGD

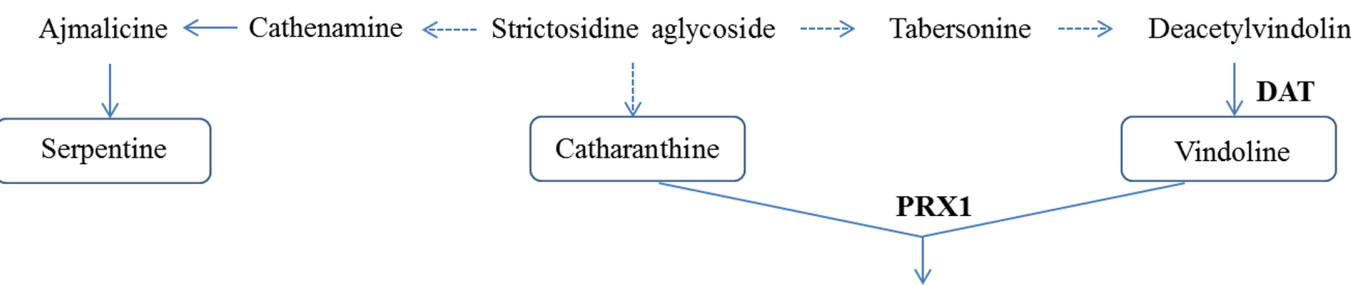

Vinblastine

Vincristine

FIGURE 5 | Seven genes (indicated in bold) which are representative in the TIAs synthetic pathway were subjected to expression analysis. G10H, geraniol 10-hydroxylase; SLS, secologanin synthase; TDC, tryptophan decarboxylase; STR, strictosidine synthase; SGD, strictosidine $\beta$-D-glucosidase; DAT, acetyl CoA: deacetylvindoline 4-O-acetyltransferase; PRX1, a-3', 4'-anhydrovinblastine synthase. Figure taken from Liu et al. (2017).

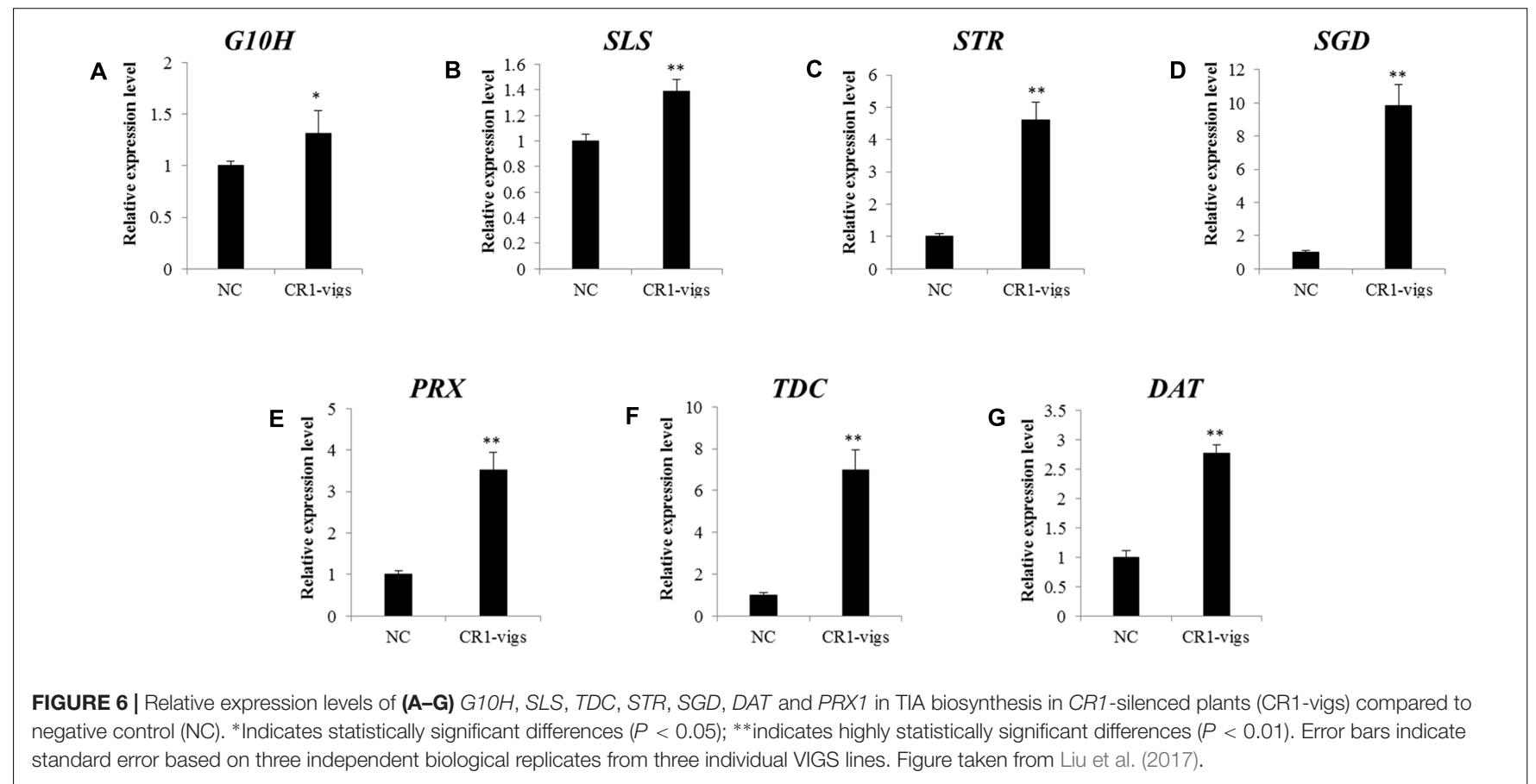




\section{A}

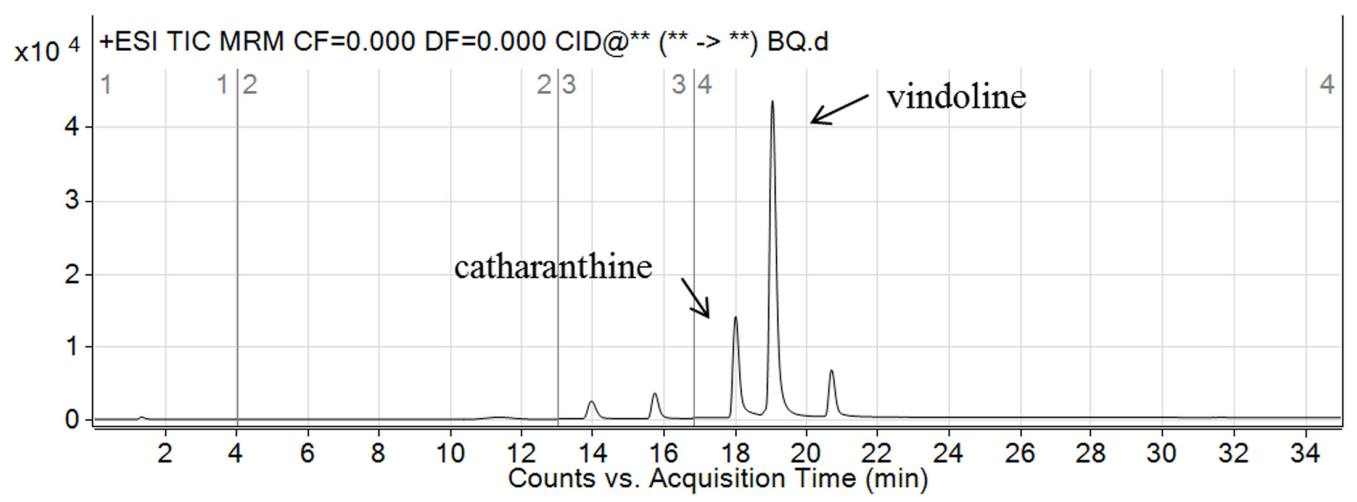

B

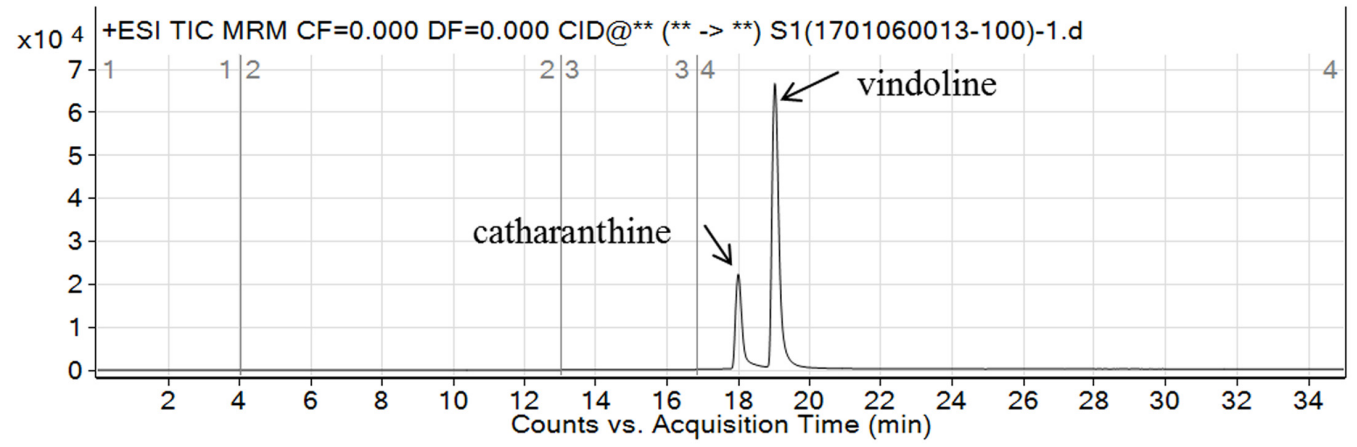

C

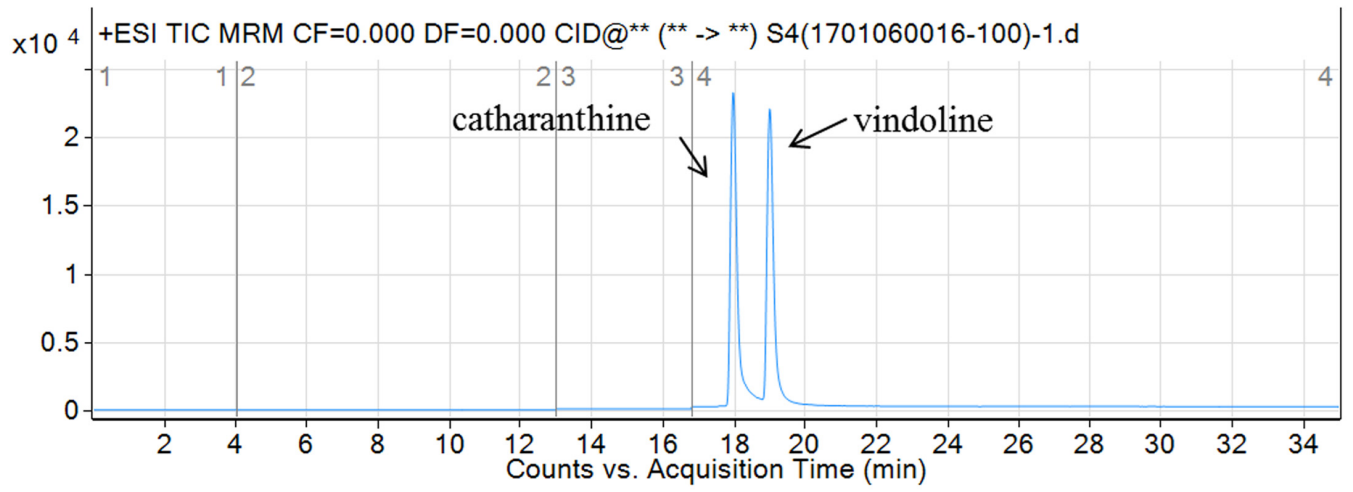

FIGURE 7 | Vindoline and catharanthine component analysis in the leaves of $C$. roseus by ESI/MS/MS. (A) ESI Tandem Mass spectrogram for the vindoline and catharanthine standard; (B) vindoline and catharanthine ion current in the leaves of CR1-silenced plants shown by ESI/MS/MS. The two peaks are vindoline (m/Z 457, RT 19.039) and catharanthine (m/z 337, RT 18.01); (C) vindoline and catharanthine ion current in the leaves of negative control shown by ESI/MS/MS. The two peaks are vindoline (m/z 457, RT 19.004) and catharanthine (m/z 337, RT 17.979). Figure taken from Liu et al. (2017).

\section{Accumulations of Vindoline and Serpentine Were Increased in CR1-Silenced Plants}

ESI/MS/MS was used to detect the catharanthine and vindoline levels; time-of-flight mass spectrometry (TOF-MS) was applied to detect the serpentine. The accumulations of catharathine and vindoline in the leaves of CR1-silenced plants were $0.191 \mathrm{nmol} / \mathrm{L}$ and $0.153 \mathrm{nmol} / \mathrm{L}$, compared to $0.198 \mathrm{nmol} / \mathrm{L}$ and $0.046 \mathrm{nmol} / \mathrm{L}$ in negative control plants (Figure 7). According to the results, silencing CR1 could increase the accumulation of vindoline, but not catharanthine. Based on the peak area of serpentine detected by TOF-MS (Figure 8), we found that the accumulation of serpentine in CR1-silenced samples was higher than that in negative control. These results are consistent with the changes of the expression levels of related genes in CR1-silenced plants, which confirmed the inference that CR1 could regulate the synthesis of TIAs with negatively regulatory function in C. roseus.

\section{DISCUSSION}

It is well known that $C$. roseus which produces approximately 130 identified TIAs is considered to be a model plant to study TIA biosynthesis. Most of the TIAs produced by C. roseus, such as ajmaline, serpentine and catharanthine, show significant clinical medical value, moreover, vinblastine and vincristine, which are 


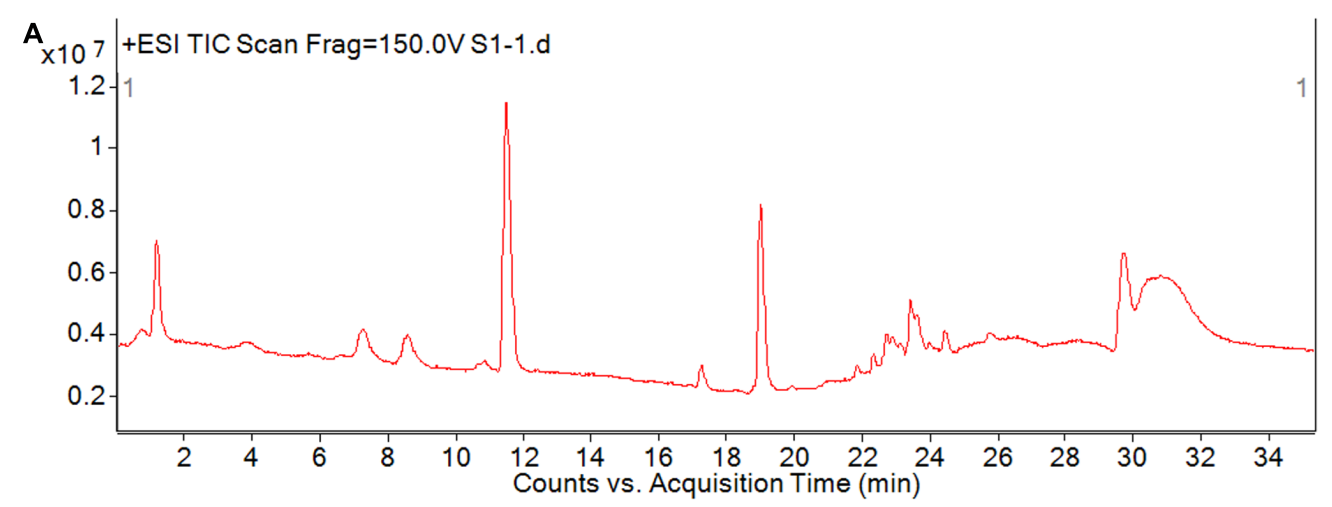

B

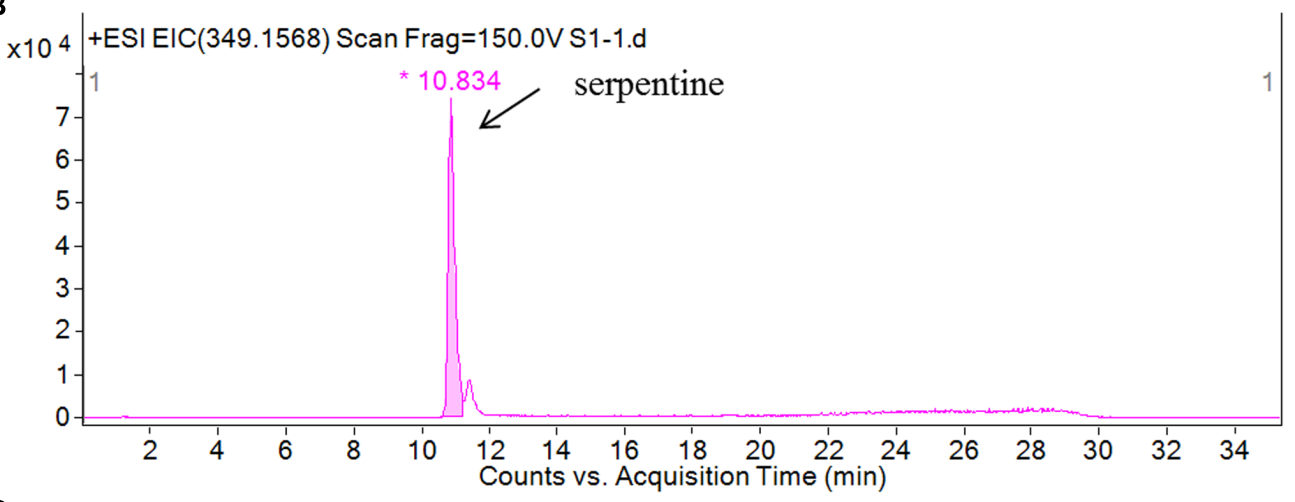

C

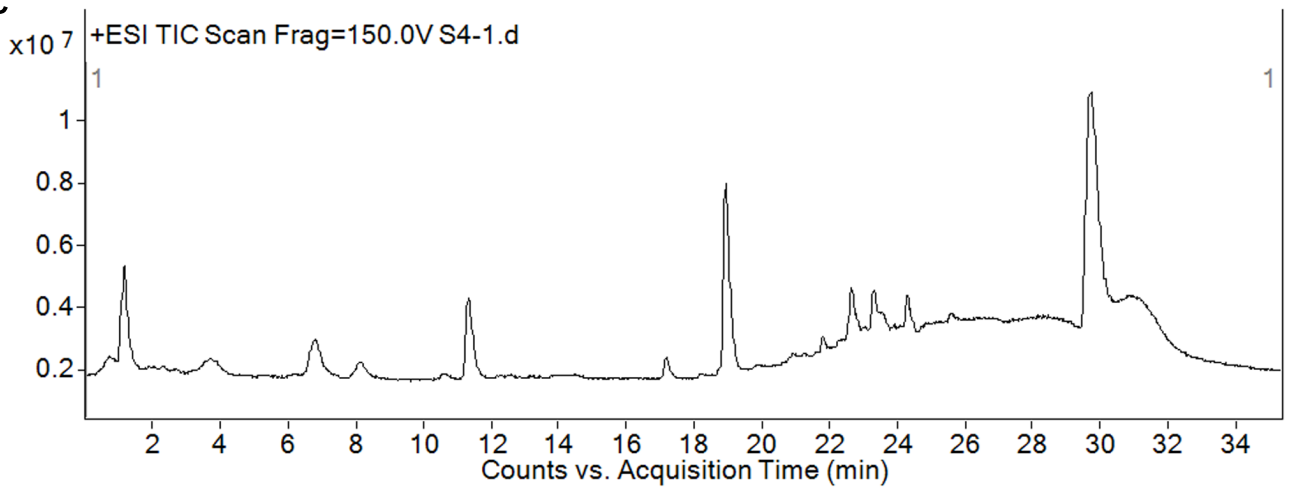

D

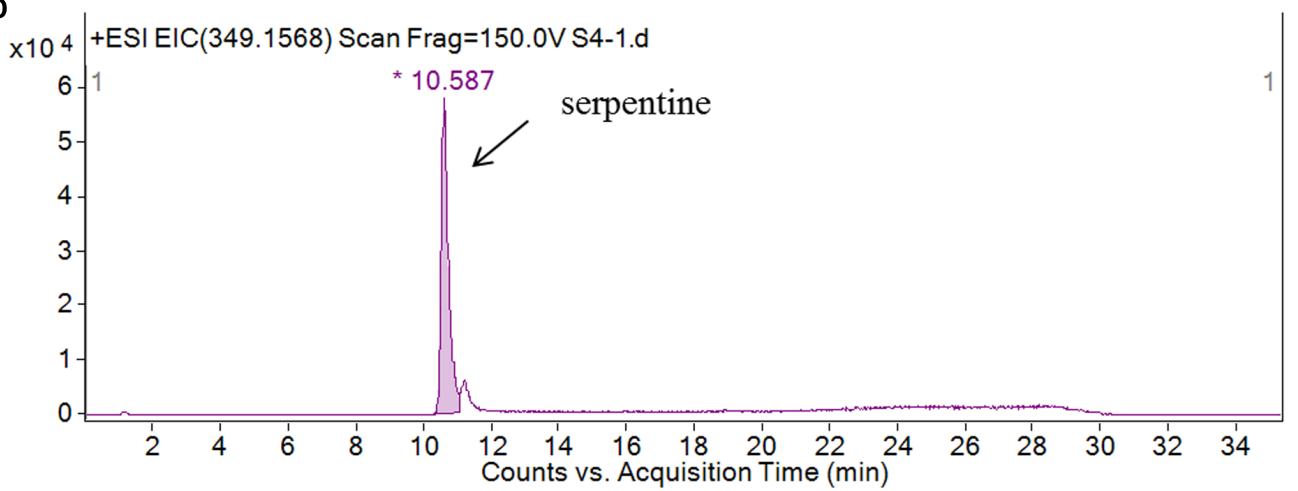

FIGURE 8 | Serpentine component analysis in the leaves of $C$. roseus by TOF/MS/MS. (A) Total ion current in the leaves of CR1-silenced plants; (B) corresponding peak of serpentine (m/z 349 RT 10. 834) component in total ion current in the leaves of CR1-silenced plants; (C) total ion current in the leaves of negative control; (D) corresponding peak of serpentine ( $\mathrm{m} / \mathrm{z} 349$ RT 10. 587) component in total ion current in the leaves of negative control. Figure taken from Liu et al. (2017). 
recognized to be the most valuable antineoplastic agents, have been widely used in the world. However, the yields of these high medical value metabolites are extremely low through traditional approach. Therefore, it is critical to find new ways which could greatly facilitate TIAs production, such as genetic engineering. Nowadays, bioinformatics has been widely used in many medicinal plants such as Dioscorea nipponica (Sun et al., 2017), Panax ginseng C. A. Meyer (Zhang et al., 2017), Taxus chinensis (Li et al., 2012; Meng et al., 2017), and Ginkgo biloba (Lin et al., 2011; He et al., 2015). High-throughput data such as transcriptome, proteome and metabolome could help us to gain an insight into the process of secondary metabolism in many non-model plants. The availability of several $C$. roseus transcriptomic databases, such as Medicinal Plant Genomics Resource (MPGR) ${ }^{1}$ (Kellner et al., 2015); PhytoMetaSyn ${ }^{2}$ (Facchini et al., 2012; Xiao et al., 2013) could greatly facilitate the studies on either biochemical pathways or transcriptional regulation in $C$. roseus. Our study utilized RNA sequencing combined with MPGR to compensate for the insufficiency of the genomic information as well as gene expression information in the discovery of candidate transcription factors involved in TIA biosynthesis regulation. By integrating bioinformatic analysis and a series of experimental methods, a novel negatively regulatory transcription factor, CR1, is identified.

The results of expression analysis of CR1-silenced plants suggested that CR1 could regulate several key genes in TIA biosynthesis, not only the genes upstream to the central precursor strictosidine which derive all the TIAs in C. roseus (G10H, SLS, TDC and STR), but the genes downstream to it (SGD, DAT and PRX1). ORCA2 and ORCA3 are the earliest found AP2/ERF transcription factors in $C$. roseus which play important roles in TIA biosynthesis. They mainly regulate the downstream genes in the seco-iridoid pathway (Menke et al., 1999; Van der Fits and Memelink, 2000; Pan et al., 2012; Li et al., 2013; Sun and Peebles, 2016), while ORCA4 is functionally overlapping but divergent with ORCA3, regulating the genes in both tryptophan pathway and seco-iridoid pathway (Paul et al., 2016). Combining these cases with our results, it can be inferred that CR1 may function together with other AP2 transcription factors as a negative feedback model to keep balance of TIA biosynthesis. Therefore,

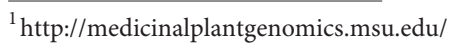

${ }^{2}$ http://www.phytometasyn.ca/index.php/

\section{REFERENCES}

Aito, K., Hirai, M. Y., and Yonekura-Sakakibara, K. (2008). Decoding genes with coexpression networks and metabolomics - Majority report by precogs. Trends Plant Sci. 13, 36-43.

Burch-Smith, T. M., Anderson, J. C., Martin, G. B., and Dinesh-Kumar, S. P. (2004). Applications and advantages of virus-induced gene silencing for gene function studies in plants. Plant J. 9, 734-746.

Chen, T., Wu, H., Wu, J., Fan, X., Li, X., and Lin, Y. (2017). Absence of Os $\beta C A 1$ causes a $\mathrm{CO} 2$ deficit and affects leaf photosynthesis and the stomatal response to CO2 in rice. Plant J. 90, 344-357. doi: 10.1111/tpj. 13497
CR1 could be used in cooperation with other AP2/ERF such as ORCA3 to further increase the accumulations of target TIAs.

The suppression of CR1 could promote the synthesis of vindoline and serpentine, but not catharathine, which may suggest that the genes in the catharathine-specific pathway can not be regulated by CR1. Vindoline and catharathine are the precursors of vinblastine and vincristine, the most valuable dimeric TIAs in $C$. roseus which have been used clinically to treat cancers since 1950s (Leveque and Jeh, 2007). If the accumulations of vindoline and catharathine are both increased, the biosynthesis of vinblastine and vincristine might be also promoted. Therefore, CR1 could be co-utilized with other transcription factors to increase the accumulations of vindoline and catharathine simultaneously. Overall, our study combined RNA sequencing and VIGS of the target gene as well as expression and metabolite analyses of silenced plants to discover an AP2/ERF transcription factor involved in TIA biosynthesis in C. roseus.

\section{AUTHOR CONTRIBUTIONS}

RW conceived and designed the experiments. JL and RW performed the experiments. RW and FG performed the data analysis. RW and JL wrote the paper. RW, FG, JR, XL, and GR revised the paper. RW secured the funds to support this research.

\section{FUNDING}

This research was supported by the National Natural Science Foundation of China.

\section{ACKNOWLEDGMENT}

The authors thank Junjun Cai (West China Hospital, Sichuan University) for language improvement.

\section{SUPPLEMENTARY MATERIAL}

The Supplementary Material for this article can be found online at: https://www.frontiersin.org/articles/10.3389/fpls.2017.02082/ full\#supplementary-material

Cloonan, N., Forrest, A. R., Kolle, G., Gardiner, B. B., Faulkner, G. J., Brown, M. K., et al. (2008). Stem cell transcriptome profiling via massive-scale mRNA sequencing. Nat. Methods 5, 613-619. doi: 10.1038/nmeth.1223

Cui, L., Ni, X., Ji, Q., Teng, X., Yang, Y., Wu, C., et al. (2015). Co-overexpression of geraniol-10-hydroxylase and strictosidine synthase improves anti-cancer drug camptothecin accumulation in Ophiorrhiza pumila. Sci. Rep. 5:8227. doi: $10.1038 /$ srep08227

De Boer, K., Tilleman, S., Pauwels, L., Vanden Bossche, R., De Sutter, V., Vanderhaeghen, R., et al. (2011). APETALA2/ETHYLENE RESPONSE FACTOR and basic helix-loop-helix tobacco transcription factors cooperatively mediate jasmonate-elicited nicotine biosynthesis. Plant J. 66, 1053-1065. doi: 10.1111/j.1365-313X.2011.04566.x 
De Geyter, N., Gholami, A., Goormachtig, S., and Goossens, A. (2012). Transcriptional machineries in jasmonate-elicited plant secondary metabolism. Trends Plant Sci. 17, 349-359. doi: 10.1016/j.tplants.2012.03.001

Dinesh-Kumar, S. P., Anandalakshmi, R., Marathe, R., Schiff, M., and Liu, Y. (2003). Virus-induced gene silencing. Methods Mol. Biol. 236, 287-294.

Facchini, P. J., Bohlmann, J., Covello, P. S., De Luca, V., Mahadevan, R., Page, J. E., et al. (2012). Synthetic biosystems for the production of high-value plant metabolites. Trends Biotechnol. 30, 127-131. doi: 10.1016/j.tibtech.2011. 10.001

Geu-Flores, F., Sherden, N. H., Courdavault, V., Burlat, V., Glenn, W. S., Wu, C., et al. (2012). An alternative route to cyclic terpenes by reductive cyclization in iridoid biosynthesis. Nature 492, 138-142. doi: 10.1038/nature11692

Guéritte, F., and Fahy, J. (2005). “The vinca alkaloids," in Anticancer Agents from Natural Products, eds G. M. L. Cragg, D. Kingston, and D. J. Newman (Boca Raton, Fla: CRC Press), 123-136.

Guo, X., Li, Y., Li, C., Luo, H., Wang, L., Qian, J., et al. (2013). Analysis of the Dendrobium officinale transcriptome reveals putative alkaloid biosynthetic genes and genetic markers. Gene 527, 131-138. doi: 10.1016/j.gene.2013.05.073

He, B., Gu, Y., Xu, M., Wang, J., Cao, F., and Xu, L. A. (2015). Transcriptome analysis of Ginkgo biloba kernels. Front. Plant Sci. 6:819. doi: 10.3389/fpls.2015. 00819

Liu, J., Cai, J., Wang, R., and Yang S. (2017). Transcriptional regulation and transport of terpenoid indole alkaloid in Catharanthus roseus: exploration of new research directions. Int. J. Mol. Sci. 18:53. doi: 10.3390/ijms18010053

Kellner, F., Kim, J., Clavijo, B. J., Hamilton, J. P., Childs, K. L., Vaillancourt, B., et al. (2015). Genome-guided investigation of plant natural product biosynthesis. Plant J. 82, 680-692. doi: 10.1111/tpj.12827

Leveque, D., and Jeh, F. (2007). Molecular pharmacokinetics of catharanthus (vinca) alkaloids. J. Clin. Pharmacol. 47, 579-588.

Li, C. Y., Leopold, A. L., Sander, G. W., Shanks, J. V., Zhao, L., and Gibson, S. I. (2013). The ORCA2 transcription factor plays a key role in regulation of the terpenoid indole alkaloid pathway. BMC Plant Biol. 13:155. doi: 10.1186/14712229-13-155

Li, S. T., Zhang, P., Zhang, M., Fu, C. H., Zhao, C. F., Dong, Y. S., et al. (2012). Transcriptional profile of Taxus chinensis cells in response to methyl jasmonate. BMC Genomics 13:295. doi: 10.1186/1471-2164-13-295

Lin, X., Zhang, J., Li, Y., Luo, H., Wu, Q., Sun, C., et al. (2011). Functional genomics of a living fossil tree. Ginkgo, based on next-generation sequencing technology. Physiol. Plant. 143, 207-218. doi: 10.1111/j.1399-3054.2011.01500.x

Liscombe, D. K., and O'Connor, S. E. (2011). A virus-induced gene silencing approach to understanding alkaloid metabolism in Catharanthus roseus. Phytochemistry 72, 1969-1977. doi: 10.1016/j.phytochem.2011. 07.001

Livak, K. J., and Schmittgen, T. D. (2001). Analysis of relative gene expression data using real-time quantitative PCR and the $2-\Delta \Delta^{C_{\mathrm{T}}}$ method. Methods 25, 402-408.

Lu, X., Zhang, L., Zhang, F., Jiang, W., Shen, Q., Zhang, L., et al. (2013). AaORA, a trichome-specific AP2/ERF transcription factor of Artemisia annua, is a positive regulator in the artemisinin biosynthetic pathway and in disease resistance to Botrytis cinerea. New Phytol. 198, 1191-1202. doi: 10.1111/nph. 12207

Meng, D., Yu, X., Ma, L., Hu, J., Liang, Y., Liu, X., et al. (2017). Transcriptomic response of Chinese yew (Taxus chinensis) to cold stress. Front. Plant Sci. 8:468. doi: $10.3389 /$ fpls.2017.00468

Menke, F. L., Champion, A., Kijne, J. W., and Memelink, J. (1999). A novel jasmonate-and elicitor-responsive element in the periwinkle secondary metabolite biosynthetic gene Str interacts with a jasmonate-and elicitorinducible AP2-domain transcription factor, ORCA2. EMBO J. 18, 4455-4463.

Montiel, G., Zarei, A., Korbes, A. P., and Memelink, J. (2011). The jasmonateresponsive element from the ORCA3 promoter from Catharanthus roseus is active in arabidopsis and is controlled by the transcription factor ATMYC2. Plant Cell Physiol. 52, 578-587. doi: 10.1093/pcp/pcr016

Mortazavi, A., Williams, B. A., McCue, K., Schaeffer, L., and Wold, B. (2008). Mapping and quantifying mammalian transcriptomes by RNA-Seq. Nat. Methods 5, 621-628. doi: 10.1038/nmeth.1226

Pan, Q., Wang, Q., Yuan, F., Xing, S., Zhao, J., Choi, Y. H., et al. (2012). Overexpression of ORCA3 and G10h in Catharanthus roseus plants regulated alkaloid biosynthesis and metabolism revealed by NMR-metabolomics. PLOS ONE 7:e43038. doi: 10.1371/journal.pone.0043038

Pandey, S. S., Singh, S., Babu, C. S., Shanker, K., Srivastava, N. K., Shukla, A. K., et al. (2016). Fungal endophytes of Catharanthus roseus enhance vindoline content by modulating structural and regulatory genes related to terpenoid indole alkaloid biosynthesis. Sci. Rep. 6:26583. doi: 10.1038/srep26583

Papon, N., Vansiri, A., Gantet, P., Chenieux, J. C., Rideau, M., and Creche, J. (2004). Histidine-containing phosphotransfer domain extinction by RNA interference turns off a cytokinin signalling circuitry in Catharanthus roseus suspension cells. FEBS Lett. 558, 85-88.

Paul, P., Singh, S. K., Patra, B., Sui, X., Pattanaik, S., and Yuan, L. (2016). A differentially regulated AP2/ERF transcription factor gene cluster acts downstream of a MAP kinase cascade to modulate terpenoid indole alkaloid biosynthesis in Catharanthus roseus. New Phytol. 213, 1107-1123. doi: 10.1111/ nph.14252

Peebles, C. A., Hughes, E. H., Shanks, J. V., and San, K. Y. (2009). Transcriptional response of the terpenoid indole alkaloid pathway to the overexpression of ORCA3 along with jasmonic acid elicitation of Catharanthus roseus hairy roots over time. Metab. Eng. 11, 76-86. doi: 10.1016/j.ymben.2008.09.002

Peebles, C. A., Sander, G. W., Hughes, E. H., Peacock, R., Shanks, J. V., and San, K. Y. (2011). The expression of 1-deoxy-D-xylulose synthase and geraniol10-hydroxylase or anthranilate synthase increases terpenoid indole alkaloid accumulation in Catharanthus roseus hairy roots. Metab. Eng. 13, 234-240. doi: 10.1016/j.ymben.2010.11.005

Runguphan, W., Maresh, J. J., and O'Connor, S. E. (2009). Silencing of tryptamine biosynthesis for production of nonnatural alkaloids in plant culture. Proc. Natl. Acad. Sci. U.S.A. 106, 13673-13678. doi: 10.1073/pnas.0903393106

Rushton, P. J., Bokowiec, M. T., Han, S., Zhang, H., Brannock, J. F., Chen, X., et al. (2008). Tobacco transcription factors: novel insights into transcriptional regulation in the Solanaceae. Plant Physiol. 147, 280-295. doi: 10.1104/pp.107. 114041

Saito, K., and Matsuda, F. (2010). Metabolomics for functional genomics, systems biology, and biotechnology. Annu. Rev. Plant Biol. 61, 463-489. doi: 10.1146/ annurev.arplant.043008.092035

Shoji, T., Kajikawa, M., and Hashimoto, T. (2010). Clustered transcription factor genes regulate nicotine biosynthesis in tobacco. Plant Cell 22, 3390-3409. doi: 10.1105/tpc.110.078543

Sun, J., and Peebles, C. A. (2016). Engineering overexpression of ORCA3 and strictosidine glucosidase in Catharanthus roseus hairy roots increases alkaloid production. Protoplasma 253, 1255-1264. doi: 10.1007/s00709-015-0881-7

Sun, W., Wang, B., Yang, J., Wang, W., Liu, A., Leng, L., et al. (2017). Weighted gene co-expression network analysis of the dioscin rich medicinal plant Dioscorea nipponica. Front. Plant Sci. 8:789. doi: 10.3389/fpls.2017.00789

Suttipanta, N., Pattanaik, S., Kulshrestha, M., Patra, B., Singh, S. K., and Yuan, L. (2011). The transcription factor CrWRKY1 positively regulates the terpenoid indole alkaloid biosynthesis in Catharanthus roseus. Plant Physiol. 157, 2081-2093. doi: 10.1104/pp.111.181834

Thagun, C., Imanishi, S., Kudo, T., Nakabayashi, R., Ohyama, K., Mori, T., et al. (2016). Jasmonate-responsive ERF transcription factors regulate steroidal glycoalkaloid biosynthesis in tomato. Plant Cell Physiol. 57, 961-975. doi: $10.1093 / \mathrm{pcp} / \mathrm{pcw} 067$

Todd, A. T., Liu, E., Polvi, S. L., Pammett, R. T., and Page, J. E. (2010). A functional genomics screen identifies diverse transcription factors that regulate alkaloid biosynthesis in Nicotiana benthamiana. Plant J. 62, 589-600. doi: 10.1111/j. 1365-313X.2010.04186.x

Udomsom, N., Rai, A., Suzuki, H., Okuyama, J., Imai, R., Mori, T., et al. (2016). Function of AP2/ERF transcription factors involved in the regulation of specialized metabolism in Ophiorrhiza pumila revealed by transcriptomics and metabolomics. Front. Plant Sci. 7:1861. doi: 10.3389/fpls.2016.01861

Van der Fits, L., and Memelink, J. (2000). ORCA3, a jasmonate-responsive transcriptional regulator of plant primary and secondary metabolism. Science 289, 295-297.

Van der Fits, L., and Memelink, J. (2001). The jasmonate-inducible AP2/ERFdomain transcription factor ORCA3 activates gene expression via interaction with a jasmonate-responsive promoter element. Plant J. 25, 43-53.

Van Der Heijden, R., Jacobs, D., Snoeijer, W., Hallard, D., and Verpoorte, R. (2004). The Catharanthus alkaloids: pharmacognosy and biotechnology. Curr. Med. Chem. 11, 607-628. 
Velásquez, A. C., Chakravarthy, S., and Martin, G. B. (2009). Virus-induced gene silencing (VIGS) in Nicotiana benthamiana and tomato. J. Vis. Exp. 10:1292. doi: $10.3791 / 1292$

Verma, M., Ghangal, R., Sharma, R., Sinha, A. K., and Jain, M. (2014). Transcriptome analysis of Catharanthus roseus for gene discovery and expression profiling. PLOS ONE 9:e103583. doi: 10.1371/journal.pone. 0103583

Wang, M., Zi, J., Zhu, J., Chen, S., Wang, P., Song, L., et al. (2016). Artemisinic acid serves as a novel ORCA3 inducer to enhance biosynthesis of terpenoid indole alkaloids in Catharanthus roseus cambial meristematic cells. Nat. Prod. Commun. 11, 715-717.

Wang, R., Lu, L., Pan, X., Hu, Z., Ling, F., Yan, Y., et al. (2015). Functional analysis of OsPGIP1 in rice sheath blight resistance. Plant Mol. Biol. 87, 181-191. doi: 10.1007/s11103-014-0269-7

Wasternack, C. (2014). Action of jasmonates in plant stress responses and development-applied aspects. Biotechnol. Adv. 32, 31-39. doi: 10.1016/j. biotechadv.2013.09.009

Wilhelm, B. T., Marguerat, S., Goodhead, I., and Bahler, J. (2010). Defining transcribed regions using RNA-seq. Nat. Protoc. 5, 255-266. doi: 10.1038/nprot. 2009.229

Xiao, M., Zhang, Y., Chen, X., Lee, E. J., Barber, C. J., Chakrabarty, R., et al. (2013). Transcriptome analysis based on next-generation sequencing of nonmodel plants producing specialized metabolites of biotechnological interest. J. Biotechnol. 166, 122-134. doi: 10.1016/j.jbiotec.2013.04.004

Yang, M., You, W., Wu, S., Fan, Z., Xu, B., Zhu, M., et al. (2017). Global transcriptome analysis of Huperzia serrata and identification of critical genes involved in the biosynthesis of huperzine A. BMC Genomics 18:245. doi: 10.1186/s12864-017-3615-8
Yang, Z., Patra, B., Li, R., Pattanaik, S., and Yuan, L. (2013). Promoter analysis reveals cis-regulatory motifs associated with the expression of the WRKY transcription factor CrWRKY1 in Catharanthus roseus. Planta 238, 1039-1049. doi: 10.1007/s00425-013-1949-2

Yu, Z. X., Li, J. X., Yang, C. Q., Hu, W. L., Wang, L. J., and Chen, X. Y. (2012). The jasmonate-responsive AP2/ERF transcription factors AaERF1 and AaERF2 positively regulate artemisinin biosynthesis in Artemisia annua L. Mol. Plant 5, 353-365. doi: $10.1093 / \mathrm{mp} / \mathrm{ssr} 087$

Zhang, H., Hedhili, S., Montiel, G., Zhang, Y., Chatel, G., Pré, M., et al. (2011). The basic helix-loop-helix transcription factor CrMYC2 controls the jasmonateresponsive expression of the ORCA genes that regulate alkaloid biosynthesis in Catharanthus roseus. Plant J. 67, 61-71. doi: 10.1111/j.1365-313X.2011. 04575.x

Zhang, J. J., Su, H., Zhang, L., Liao, B. S., Xiao, S. M., Dong, L. L., et al. (2017). Comprehensive characterization for ginsenosides biosynthesis in ginseng root by integration analysis of chemical and transcriptome. Molecules 22:E889. doi: 10.3390/molecules22060889

Conflict of Interest Statement: The authors declare that the research was conducted in the absence of any commercial or financial relationships that could be construed as a potential conflict of interest.

Copyright (c) $2017 \mathrm{Liu}, \mathrm{Gao}, \mathrm{Ren}, \mathrm{Lu}$, Ren and Wang. This is an open-access article distributed under the terms of the Creative Commons Attribution License (CC BY). The use, distribution or reproduction in other forums is permitted, provided the original author(s) or licensor are credited and that the original publication in this journal is cited, in accordance with accepted academic practice. No use, distribution or reproduction is permitted which does not comply with these terms. 